\title{
Perceived impact of the COVID-19 pandemic on child and adolescent psychiatric services after 1 year (February/March 2021): ESCAP CovCAP survey
}

\author{
Alexis Revet ${ }^{1,2}$ (D) Johannes Hebebrand ${ }^{3} \cdot$ Dimitris Anagnostopoulos ${ }^{4,5} \cdot$ Laura A. Kehoe $^{6} \cdot$ Gertraud Gradl-Dietsch $^{3}$. \\ COVID-19 Child and Adolescent Psychiatry Consortium • Paul Klauser ${ }^{7,8}$
}

Received: 13 July 2021 / Accepted: 22 July 2021 / Published online: 29 July 2021

(c) Springer-Verlag GmbH Germany, part of Springer Nature 2021

\begin{abstract}
In April 2020, the European Society for Child and Adolescent Psychiatry (ESCAP) Research Academy and the ESCAP Board launched the first questionnaire of the CovCAP longitudinal survey to estimate the impact of COVID-19 on child and adolescent psychiatry (CAP) services in Europe. In this brief report, we present the main findings from the second questionnaire of the survey, one year after the COVID-19 pandemic began to hit Europe (i.e., February/March 2021). While service delivery to patients and their families was affected in a major way (reported by 68\%) at the beginning of the pandemic, the majority of respondents (59\%) in this second survey only reported a minor impact on care delivery. The use of telemedicine remained widespread (91\%) but the proportion of CAP services partially closed or transformed to accommodate COVID-19 patients (59\% in 2020) dropped to 20\%. On the other hand, the perceived impact on the mental health and psychopathology of children and adolescents dramatically increased from "medium" (> 50\%) in 2020 to "strong" or "extreme" (80\%) in 2021. Four nosographic entities were particularly impacted: suicidal crises, anxiety disorders, eating disorders and major depressive episodes. Accordingly, this was associated with a substantial increase in the number of referrals or requests for assessments (91\% reported an increase in 2021 while $61 \%$ reported a decrease in 2020). Finally, heads of the CAP departments expressed strong concerns regarding the management of the long-term consequences of this crisis, especially regarding the provision of care in light of the perceived increase in referrals.
\end{abstract}

Keywords COVID-19 $\cdot$ Child and adolescent psychiatry $\cdot$ Europe $\cdot$ Mental health $\cdot$ Children $\cdot$ Adolescents

The COVID-19 pandemic and the measures taken to control its spread have wreaked havoc worldwide, resulting in

The members of the COVID-19 Child and Adolescent Psychiatry Consortium are mentioned in the Acknowledgments section.

Paul Klauser

paul.klauser@chuv.ch

1 Service Universitaire de Psychiatrie de L'Enfant et de L'Adolescent, CHU de Toulouse, Toulouse, France

2 CERPOP, Université de Toulouse, Inserm, UPS, Toulouse, France

3 Department of Child and Adolescent Psychiatry, Psychosomatics and Psychotherapy, LVR Klinikum Essen, University Hospital Essen, University of Duisburg-Essen, Essen, Germany

4 Medical School, National and Kapodistrian University of Athens, Athens, Greece more than 3 million deaths and forcing billions of people into isolation, profoundly affecting all aspects of daily life. Numerous studies have reported on the consequences of the COVID-19 crisis on mental health [1], but few have focused on children and adolescents [2,3].

5 President of European Society for Child Adolescent Psychiatry, National \& Kapodistrian University of Athens, Athens, Greece

6 Medical Communications, Avenue des Cadolles 12D, 2000 Neuchatel, Switzerland

7 Service of Child and Adolescent Psychiatry, Department of Psychiatry, Lausanne University Hospital, Lausanne, Switzerland

8 Center for Psychiatric Neuroscience, Department of Psychiatry, Lausanne University Hospital, Lausanne, Switzerland 
In April 2020, the European Society for Child and Adolescent Psychiatry (ESCAP) Research Academy, a network of young clinician-scientists in child and adolescent psychiatry (CAP) [4], and the ESCAP Board launched the CovCAP longitudinal survey to estimate the impact of COVID-19 on CAP services in Europe and to assess their ability to meet the new challenges brought on by the crisis. Heads of CAP university services in ESCAP member countries were contacted by email and invited to complete a self-report online questionnaire survey (detail of the methods have been published previously [5]; some questions were modified, removed or added in the second survey of the CovCAP study). The first stage of the study (i.e., March/April 2020) yielded surprisingly consistent results, showing a substantial reduction in the number of both outpatient visits and hospital admissions and a moderate effect on psychopathology. In contrast, the effect on the organization of CAP services appeared profound, with a clear acceleration in the adoption of new technologies, including telepsychiatry [5]. In this brief report, we briefly present the main findings from the second step of the survey, 1 year after the COVID-19 pandemic began to hit Europe (i.e., February/March 2021).

The survey was distributed to 266 heads of CAP university services in 32 countries by email between February 19th and March 25th, 2021. In total, 72 responded (27.1\%), among which, 60 filled in the questionnaire completely (first and last response received: 19 February and 28 March 2021, respectively). Twenty-two countries were represented, among which, Germany had the highest number of responses (12 respondents; 16.7\%). We did not obtain replies from Albania, Cyprus, Finland, Iceland, Israel, Luxemburg, Norway, Romania, Serbia and Sweden.

A selection of answers with a comparison between the two time periods (if possible) is presented in Table 1. The full data set is available on request (paul.klauser@chuv. ch). In general, the overall perception of the impact of the crisis on the mental health of children and adolescents has changed markedly between the two study periods, from an impact mainly ( $>50 \%$ ) perceived as "medium" in April/May 2020 to an impact perceived as "strong" or "extreme" by 80\% of respondents in February/March 2021 (Fig. 1a). In comparison to before the pandemic, while $61 \%$ of respondents reported a decrease in referrals or requests for assessment in March/April 2020, 91\% of respondents reported an increase in February/March 2021. Accordingly, the impact on psychopathology appeared much more marked during this second phase of the study, with four nosographic entities particularly impacted: suicidal crises (83\%), anxiety disorders $(70 \%)$, eating disorders (64\%) and major depressive episodes (61\%) (Fig. 1b). Similarly, the pandemic seems to influence the psychopathology patients present with (e.g., fear of contamination) in a higher proportion of patients in February/March 2021 than in April/May 2020 (Fig. 1c).
Not surprisingly, the mental health determinant most frequently reported for both children and adolescents was school closure. However, there are differences in the other factors: parental stress appears to be the second most important determinant for children, while for adolescents, home confinement and assembly restrictions are more frequently reported (Fig. 1d).

While the provision of services to patients and their families was initially affected to a major degree (affirmed by $68 \%$ of respondents in 2020), the majority of respondents (59\%) reported only a minor impact on care provision in this second CovCAP survey. The use of telemedicine has remained important (91\%), but guidelines to accompany its usage did not generalize (35\% in 2020; $40 \%$ in 2021). Specific measures to support bereaved children to deal with COVID-19-related grief or trauma have not become widespread either (16\% in 2020; $20 \%$ in 2021). While $59 \%$ of respondents had a portion of their services closed or transformed to accommodate COVID-19 patients in April/May 2020, this proportion dropped to 20\% in February/March 2021. Respondents' major pandemic-related service delivery concerns for this second phase of the survey were not being able to maintain specific treatment groups for distinct groups of patients $(70 \%)$, not being in sufficient contact with patients and families in need (60\%), and having to manage the absence of some of their staff (33\%).

Although the perception of their team's mental readiness to handle this crisis did not really change from April/May 2020 to February/March 2021 (60-63, respectively, on a scale from 0 to 100), a subjective improvement was noted between the two study periods in terms of the equipment of the services' infrastructures to manage COVID-positive patients (42 in April/May 2020 to 57 in February/March 2021, on a scale of 0-100). Interestingly, the level of acceptance of COVID-19 vaccination was perceived as high in team members of nearly three quarters of respondents and $23 \%$ of them reported a vaccination rate higher than $50 \%$ in their respective services.

Respondents' major concerns related to CAP for when the pandemic is over were difficulties in managing the increased numbers of patients $(83 \%)$, difficulties in managing families in situation of high psychosocial precariousness $(83 \%)$ and reduced financial resources for CAP services (48\%).

Analysis of the free comments section of the questionnaire confirmed the contrast between a reduced activity in CAP services at the beginning of the pandemic in Europe and a dramatic increase in referrals for assessment and/or hospitalization as of the end of 2020 . The impact on young people's schooling as well as an increased incidence of anxiety, depression, self-harm and suicide attempts were also highlighted. While high levels of stress and even feelings of burnout among care teams were reported, some respondents also emphasized the effectiveness of certain 
Table 1 Most relevant results from the second stage (February/March 2021) of the ESCAP CovCAP survey

\section{Screening for COVID-19 in your CAP service}

How rapidly can you obtain a COVID-19 test result for an inpatient?

$(n=61$ in April-May 2020; $n=56$ in February-March 2021; $n$ [\%])

We have no experience, no inpatient has been tested by us or upon our request

$\begin{array}{ll}7(11.5) & 1(1.8) \\ - & 31(55.4) \\ 37(60.7) & 21(37.5) \\ 14(23) & 3(5.4) \\ 2(3.3) & 0 \\ 1(1.6) & 0\end{array}$

Within a few hours

Within $24 \mathrm{~h}$

24-48 h

$1(1.6)$

0

\section{Psychopathology in your CAP service}

How do you perceive the impact of the COVID-19 pandemic on school dropout (interruption of schooling, independently of lockdown or school closure) of children and adolescents in your region? $(n=64 ; n[\%])$

Negligible

Low

Medium

Strong

Extreme

Currently, and in comparison with early 2020, prior to the beginning of the pandemic, are you seeing changes in the number of referrals or requests for assessments? ( $n=64$ in April-May 2020; $n=64$ in February-March 2021; $n$ [\%])

No

Yes, a decline

Yes, an increase

Has a patient been treated in your services whose mental symptoms seemingly directly resulted from a COVID-19 infection (post-infectious mental disorder)? $(n=64 ; n[\%])$

I am not aware of a single patient

Yes, a single case

Yes, several cases

Treatment delivery in your CAP service

Are your physicians/psychologists using telemedicine to reduce the number of personal contacts with patients and family members $(n=60$ in April-May 2020; $n=64$ in Febru$\operatorname{ary}-$ March 2021; $n$ [\%])

Yes

No

Are you using guidelines for telemedicine? ( $n=60$ in April-May 2020; $n=62$ in February-March 2021; $n$ [\%])

Yes

No

Have you established a specific procedure (e.g., videoconferences) to support bereaved children to deal with COVID-19-related grief or trauma? ( $n=61$ in April-May 2020; $n=64$ in February-March 2021; $n$ [\%])

Yes

No

Provisions of your CAP service

How are the currently imposed regulatory measures affecting your clinical services? (more than one may apply) $(n=56 ; n[\%])$

Closure of day-care units

Restriction/reduction of the number of day-care patients

Closure of specific inpatient wards/units

$-$

Restriction/reduction of the number of inpatients 
Table 1 (continued)

Restricted access to outpatient units (e.g., for medically relevant cases or emergencies
only)

To what extent is the pandemic currently affecting the provisions of services to patients and their families? ( $n=59$ in April-May 2020; $n=63$ in February-March 2021; $n$ [\%])

Not at all

To a minor degree only

To a major degree

Extremely

Since the 1st of January 2021, has any part of your service been closed or transformed for emergency COVID-19 use? ( $n=59$ in April-May 2020; $n=63$ in February-March $2021 ; n[\%])$

Yes

No

If you can, please indicate the average daily number of inpatients (beds) (pertains to estimated average number of outpatients per day in 2019 irrespective of initial referrals or recontacts) at your hospital

Please provide today's numbers for inpatients (number of beds occupied). If you are filling the questionnaire during the weekend, estimate the average occupancy during the last week ( $n=42$ in April-Mai 2020)

If you can, please indicate the average daily number of inpatients (beds) (pertains to estimated average number of inpatients per day in 2019 irrespective of initial referrals or recontacts) at your hospital

Please provide the daily average number of inpatients (number of beds occupied each day on average) during the previous week ( $n=43$ in February-March 2021)

Changes inpatients ${ }^{\mathrm{c}}(n[\%])$

No

Decrease

Increase

If you can, please indicate the average daily number of outpatients (pertains to estimated average number of outpatients per day in 2019 irrespective of initial referrals or recontacts) at your hospital

Please provide today's numbers for outpatients (outpatient visits). If you are filling the questionnaire during the weekend, estimate the average occupancy during the last week $(n=46$ in April-Mai 2020)

If you can, please indicate the average daily number of outpatients (pertains to estimated average number of outpatients per day in 2019 irrespective of initial referrals or recontacts) at your hospital

Please provide the daily average number of outpatients (outpatients visits each day on average) during the previous week ( $n=48$ in February-March 2021)

Changes outpatients ${ }^{\mathrm{c}}(n[\%])$

No

Decrease

Increase

What are your current major concerns related to the pandemic with regard to service provisions? (more than one answer may apply) $(n=63 ; n[\%])$

We are not sufficiently in contact with patients and families in need

We are not able to maintain specific therapeutic groups for distinct groups of patients

Members of my team are not as attentive as usual to their patient

Many employees are absent

Meetings to manage the crisis prevent me from doing my work

We are running a deficit, because we treat too few patients

Other

$-$

April-May 2020a

February-March 2021

$22(39.3)$

2 (3.3)

$10(16.9)$

$40(67.8)$

$7(11.9)$

b

See Changes inpatients ${ }^{c}$

See Changes inpatients ${ }^{c}$

$-$

$6(14.3)$

33 (78.6)

3 (7.1)

See Changes outpatients ${ }^{c}$

See Changes outpatients ${ }^{c}$

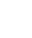

See Changes outpatients ${ }^{\mathrm{c}}$

See Changes outpatients ${ }^{c}$

$34(82.9)$

$21(43.8)$

$4(9.8)$

$10(20.8)$

\begin{tabular}{ll}
- & $38(60.3)$ \\
- & $44(69.8)$ \\
- & $5(7.9)$ \\
- & $21(33.3)$ \\
- & $16(25.4)$ \\
- & $9(14.3)$ \\
- & $13(20.6)$ \\
\hline
\end{tabular}


Table 1 (continued)

April-May $2020^{\mathrm{a}}$

February-March 2021

\section{Your CAP team}

How well was your team mentally equipped to handle COVID-19-positive patients during the last 3 months? ( $n=57$ in April-May 2020; $n=59$ in February-March 2021; mean $\pm \mathrm{SD}$ )

How well was the infrastructure of your service equipped to handle COVID-19 positive patients during the last 3 months? ( $n=56$ in April-May 2020; $n=58$ in FebruaryMarch 2021; mean \pm SD)

How do you perceive the level of COVID-19 vaccine acceptance among your team? $(n=61 ; n[\%])$

High

Medium

$6.4 \pm 11.7$

$63.47 \pm 19.99$

Low

$42.4 \pm 35.9$

$57.34 \pm 24.06$

Which proportion of your team members have already received a first dose of a COVID19 vaccine? $(n=61 ; \mathrm{n}[\%])$

$0 \%$

$0.1-4.9 \%$

$5-9.9 \%$

$10-24.9 \%$

$25-49.9 \%$

$50-74.9 \%$

$>75 \%$

$\begin{array}{ll}- & 45(73.8) \\ - & 14(23) \\ - & 2(3.3) \\ & \\ - & 2(3.3) \\ - & 4(6.6) \\ - & 2(3.3) \\ - & 8(13.1) \\ - & 3(4.9) \\ - & 14(23) \\ - & 28(45.9)\end{array}$

\section{After the COVID-19 crisis}

What are your major concerns related to CAP for when the pandemic is over? (more than one answer may apply) $(n=63 ; n[\%])$

Reduced financial resources for CAP services

Difficulties in managing the increased numbers of patients

Difficulties in managing families in situation of high psychosocial precariousness

Difficulties in managing post-infection symptoms affecting mental health

$-\quad 29(48)$

$-\quad 50(83)$

$-\quad 50(83)$

Difficulties in reopening some units in CAP services

$-\quad 9(15)$

Other

$-\quad 10(17)$

$-\quad 4(7)$

$C A P$ child and adolescent psychiatry, $S D=$ standard deviation

${ }^{\text {a }}$ Some questions were modified, removed or added in the second questionnaire of the CovCAP study. In the "April-May 2020" column, only answers to questions that were strictly identical between the two stages of the study are presented

${ }^{b}$ For the first stage of the CovCAP study (April/May 2020), the title of the question was: "Has any part of your service been closed or transformed for emergency COVID-19 use?"

${ }^{\mathrm{c}}$ Data were recoded to measure for each respondent longitudinal changes (from the pre-pandemic period) at each time point

stress management and coping techniques and the fantastic achievement of their teams in terms of adaptability and flexibility. Finally, concerns about the future of young people's mental health also emerged, particularly for very young children and children from low socio-economic backgrounds, which should lead, according to some heads of CAP service, to strong political support for mental health policies in Europe. The complete free comments are available as supplementary material.

As previously highlighted [5], this study is not without limitations. First, the overall response rate was significantly lower than in the first part of the survey $(27 \%$ vs. $50 \%$ ), and only 22 countries were eventually represented, despite sending 4 reminders in total. This severely restricted the scope of our results and the possibilities of comparison between countries. The lower response rate probably reflects a degree of weariness on the part of heads of department in the face of the multiplication of questionnaire studies, but it could also represent the intensity of their workload in this period of crisis. Second, the respondents in both surveys only partially overlap. Hence, comparisons between the two time points provided in the text and the table are based on cross-sectional data and should be interpreted accordingly. Finally, most of the questions were subject to personal interpretation and some answers also required rough estimates, which means that the data collected represent only general impressions of the heads of service. 

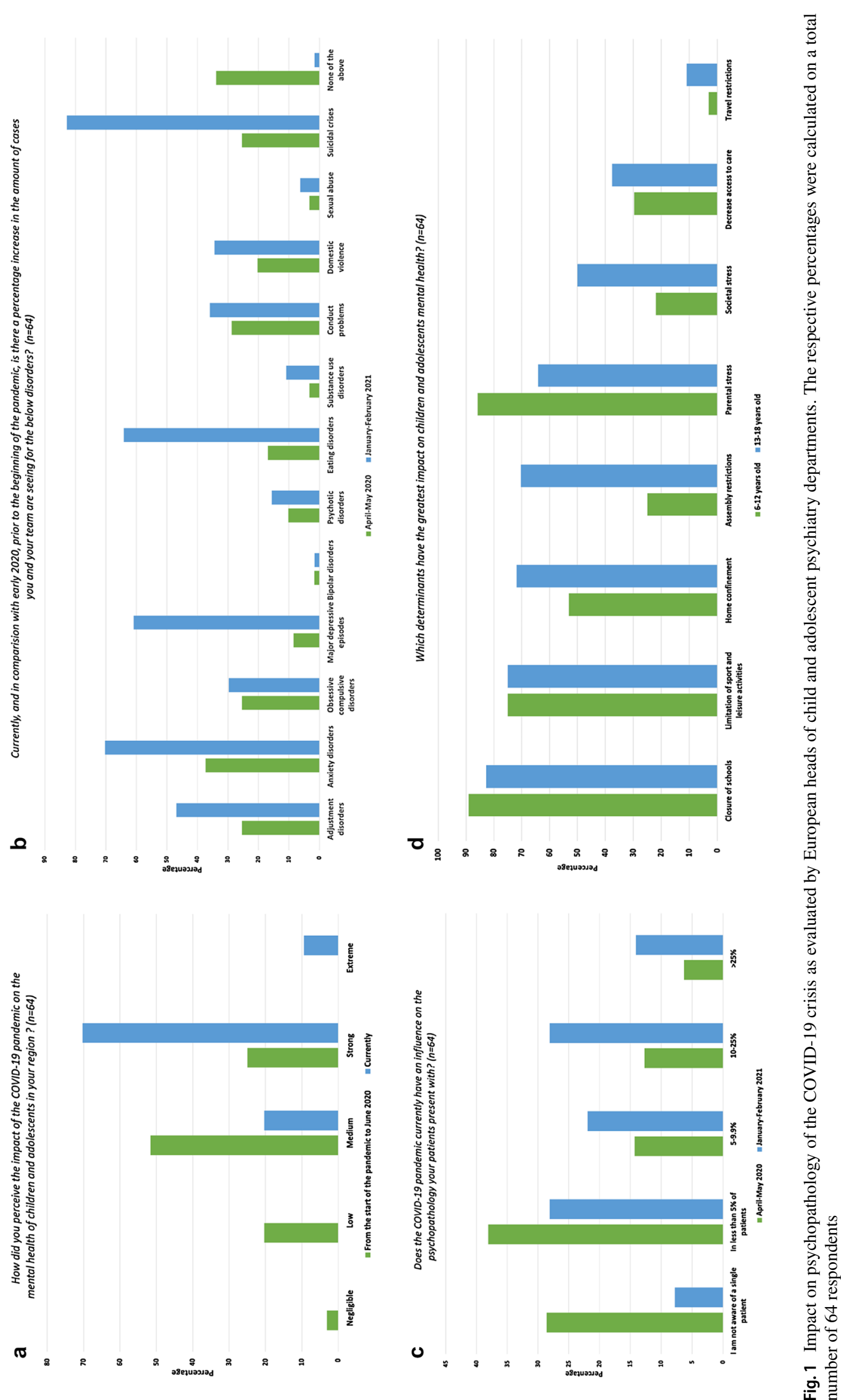
On the one hand, the results of this second phase of the CovCAP study show a more moderate effect after 1 year of the pandemic on the organization of CAP services, with the implementation of structural adjustments and the continued use of new technologies, notably telepsychiatry. On the other hand, the perceived impact on the mental health and psychopathology of children and adolescents seems major and associated to a very substantial increase in the number of referrals or requests for assessments. Finally, heads of the CAP departments expressed strong concerns regarding the management of the long-term consequences of this crisis, especially regarding the provision of care in light of the perceived increase in referrals.

Supplementary Information The online version contains supplementary material available at https://doi.org/10.1007/s00787-021-01851-1.

Acknowledgements The authors would like to thank the members of the ESCAP Board who gave their precious feedback on the first version of the questionnaire. They also would like to thank all the respondents to this study who took the time to complete the questionnaire. PK is supported by a Fellowship from the Adrian and Simone Frutiger Foundation. We thank the Center for Clinical Research at CHUV for the provision of the REDCap ${ }^{\circledR}$ platform and their logistical support.

Members of the COVID-19 Child and Adolescent Psychiatry Consortium: Marija Anderluh: Child Psychiatry Unit, University Children's Hospital, University Medical Centre, Ljubljana, Slovenia; Marco Armando: Service of Child and Adolescent Psychiatry, Department of Psychiatry, Lausanne University Hospital, Lausanne, Switzerland; Florence Askenazy: CoBTek, Université Côte d'Azur (UCA), Service Universitaire de Psychiatrie de l'Enfant et de l'Adolescent, CHU Lenval, Nice, France; Tobias Banaschewski: Child and Adolescent Psychiatry and Psychotherapy, Central Institute of Mental Health, Medical Faculty Mannheim, University of Heidelberg, Mannheim, Germany; Stephan Bender: Department of Child and Adolescent Psychiatry, Psychsomatics and Psychotherapy, University Hospital Cologne and Medical Faculty, University of Cologne, Cologne, Germany; Angelo Bernardon: Klinik für Kinder-und Jugendpsychiatrie und Psychotherapie Königsfelderstrasse 1, 5210, Windisch, Switzerland; Romuald Brunner: Clinic for Child and Adolescent Psychiatry, Psychosomatics and Psychotherapy, University of Regensburg, Regensburg, Germany; Samuele Cortese: University of Southampton, Southampton, United Kingdom; Richard Delorme: Child \& Adolescent Psychiatry Department, Robert Debré Hospital, APHP, Paris, France; Peter Deschamps: Department of Psychiatry, University Medical Centre, Utrecht, The Netherlands; Katarina Dodig-Ćurković: Unit for Child and Adolescent Psychiatry, University Health Center, Osijek, Croatia and Faculty for Health and Dental Medicine, School of Medicine, Osijek, Croatia; Maja Drobnic Radobuljac: University of Ljubljana, School of Medicine, Vrazov trg 2, Ljubljana, Slovenia; Bernadka Dubicka: University of Manchester and Pennine Care Foundation Trust, Manchester, United Kingdom; Mette Falkenberg Krantz: CORE-Copenhagen Research Centre for Mental Health, Mental Health Center Copenhagen, Capital Region of Denmark, Copenhagen University Hospital, Hellerup, Denmark; Arnaud Fernandez: CoBTek, Université Côte d'Azur (UCA), Service Universitaire de Psychiatrie de l'enfant et de l'adolescent, CHU Lenval, Nice, France; Vanessa Fonseca Pinto: CHUV, Lausanne, Switzerland; Tomislav Franic: University of Split School of Medicine, Department of Psychiatry, Split, Croatia; Oliver Fricke, Child and Adolescent Psychiatry, Witten/Herdecke University and Gemeinschaftskrankenhaus Herdecke, Herdecke, Germany; Priscille Gerardin: Hôpital Charles Nicolle, CHU Rouen and CH Rouvray, 76000 Rouen,
France; Morgane Gindt: Hôpitaux Pédiatriques de Nice, CHU Lenval, Nice, France; Hojka Gregorič Kumperščak: University Medical Centre Maribor, Maribor, Slovenia; Teresa Goldschmidt: Centro Hospitalar Universitário Lisboa Norte, Hospital de Santa Maria Avenida Professor Egas Moniz, Lisboa, Portugal; Beate Herpertz-Dahlmann: Department of Child and Adolescent Psychiatry and Psychotherapy of the RWTH, Aachen, Germany; Carole Kapp: Service of Child and Adolescent Psychiatry, Department of Psychiatry, Lausanne University Hospital, Lausanne, Switzerland; Konstantinos Kotsis: Department of Psychiatry, University of Ioannina, Ioannina, Greece; Luisa Lázaro: Department of Child and Adolescent Psychiatry and Psychology, Hospital Clínic, IBIDAPS, CIBERSAM, University of Barcelona, Barcelona, Spain; Lucia Margari: University of Bari "Aldo Moro", Bari, Italy; Francesco Margari: University of Bari "Aldo Moro", Bari, Italy; Luigi Mazzone: Child Neurology and Psychiatry Unit, Systems Medicine Department, University of Rome Tor Vergata, Via Montpellier 1, 00133 Rome, Italy; Alda Mira Coelho: Serviço de Psiquiatria de Infância e Adolescência, Centro Hospitalar e Universitário São João, Porto, Portugal; Eva Möhler: Saarland University Hospital, Homburg, Germany; Gonca Ozyurt: Katip Çelebi University Medical School Child and Adolescent Psychiatry Department, Izmir, Turkey; Montserrat Pamias Massana: Hospital Parc Taulí Sabadell, UAB-CIBERSAM, Barcelona, Spain; Adriana Pastore: Azienda Ospedaliero-Universitaria Consorziale Policlinico, Dipartimento di Scienze Mediche di Base, Neuroscienze ed Organi di Senso, Università degli Studi di Bari "Aldo Moro", Bari, Italy; Bea Pàszthy: Semmelweis University, 1st Department of Paediatrics, Budapest, Hungary; Ana Sofia Pereira da Rocha: Service of Child and Adolescent Psychiatry, Department of Psychiatry, Lausanne University Hospital, Lausanne, Switzerland; Carla Pinho: Department of Child and Adolescent Psychiatry, Centro Universitário de Coimbra, Coimbra, Portugal; Paul Plener: Department of Child and Adolescent Psychiatry, Medical University Vienna, Vienna, Austria; Jiri Podlipny: Department of Psychiatry, Charles University, Faculty of Medicine and University Hospital in Plzen, Plzen, Czech Republic; Nadia Polnareva: Clinic of Child Psychatry "St. Nicola", University Hospital Alexandrovska 1, G. Sofiiski str. Sofia 1431, Sofia, Bulgaria; Luise Poustka: Klinik für Kinder und Jugendpsychiatrie/Psychotherapie, Universitätsmedizin Göttingen, Göttingen, Germany; Diane Purper-Ouakil: CHU Montpellier-Saint Eloi, Child and Adolescent Psychiatry (MPEA1), Montpellier, France; Marta Rapado-Castro: Department of Child and Adolescent Psychiatry, Institute of Psychiatry and Mental Health, Hospital General Universitario Gregorio Marañón, School of Medicine, Universidad Complutense, IiSGM, CIBERSAM, Madrid, Spain; Asilay Seker: Cambridgeshire and Peterborough NHS Foundation Trust, Cambridge, United Kingdom; Aspasia Serdari: Department of Child and Adolescent Psychiatry, Medical School, Democritus University of Thrace, University Hospital of Alexandroupolis, Alexandroupolis, Greece; Tetiana Skrypnyk: Research Institute of Psychiatry of the Ministry of Health of Ukraine, Department of Mental Disorders of Children and Adolescents, Kyiv, Ukraine; Paloma Torres: Hôpital PitiéSalpêtrière, Service de Pédopsychiatrie, Paris France; Jana Trebaticka: Department of Paediatric Psychiatry, Faculty of Medicine Comenius University, The National Institute of Children's Diseases, Bratislava, Slovakia; Kerstin von Plessen: Service of Child and Adolescent Psychiatry, Department of Psychiatry, Lausanne University Hospital, Lausanne, Switzerland; Susanne Walitza: Department of Child and Adolescent Psychiatry and Psychotherapy of the Psychiatric University Hospital Zurich, University Zurich, Switzerland; Nihal Yurteri: Department of Child and Adolescent Psychiatry, Faculty of Medicine, Düzce University, Düzce, Turkey; Florian Daniel Zepf: Department of Child and Adolescent Psychiatry, Psychosomatic Medicine and Psychotherapy, Jena University Hospital, Friedrich Schiller University Jena, Jena, Germany. 
Author contributions AR, PK, LK and JH designed the study and wrote the protocol. JH, AR and PK designed the first version of the questionnaire, which was then corrected and improved by LK, DA and members of the Board of the European Society for Child and Adolescent Psychiatry (ESCAP). JH, GGD, AR, PK and LK designed the second version of the questionnaire. AR and PK conducted the analyses and produced the first draft of the manuscript. JH, LK, GGD, and DA contributed to the interpretation of the data, drafted portions and substantively revised all drafts of the manuscript for important intellectual content. All authors contributed to and have approved the final manuscript. AR and PK take responsibility for the integrity of the data and the accuracy of the data analysis.

\section{Declarations}

Conflict of interest The authors have no conflict of interest to declare related to this study.

Ethical approval Ethics committee approval was not required for this anonymous web-based questionnaire study.

\section{References}

1. Gloster AT, Lamnisos D, Lubenko J et al (2020) Impact of COVID-19 pandemic on mental health: An international study.
PLoS ONE 15:e0244809. https://doi.org/10.1371/journal.pone. 0244809

2. Wang G, Zhang Y, Zhao J et al (2020) Mitigate the effects of home confinement on children during the COVID-19 outbreak. Lancet 395:945-947. https://doi.org/10.1016/S0140-6736(20)30547-X

3. Ford T, John A, Gunnell D (2021) Mental health of children and young people during pandemic. BMJ 372:n614. https://doi.org/ 10.1136/bmj.n614

4. Revet A, Hebebrand J, Bhide S et al (2018) Dual training as clinician-scientist in child and adolescent psychiatry: are we there yet? Eur Child Adolesc Psychiatry 27:263-265. https://doi.org/ 10.1007/s00787-017-1104-x

5. Revet A, Hebebrand J, Anagnostopoulos D et al (2021) ESCAP CovCAP survey of heads of academic departments to assess the perceived initial (April/May 2020) impact of the COVID-19 pandemic on child and adolescent psychiatry services. Eur Child Adolesc Psychiatry. https://doi.org/10.1007/s00787-020-01699-x 